\title{
Uma revisão das atividades biológicas da trans-desidrocrotonina, um produto natural obtido de Croton cajucara
}

\author{
Marcília Pinheiro da Costa ${ }^{1}$, Nereide Stela Santos Magalhães ${ }^{1}$, Fabiano E. S. Gomes², \\ Maria Aparecida M. Maciel $^{2 *}$
}

\author{
${ }^{1}$ Departamento de Bioquímica, Laboratório de Imunopatologia Keizo-Asami (LIKA), Universidade Federal de \\ Pernambuco, Cidade Universitária, 50670-901, Recife, PE, Brasil, \\ ${ }^{2}$ Departamento de Química, Universidade Federal do Rio Grande do Norte, Campus Universitário, \\ 59078-970, Natal, RN, Brasil
}

\begin{abstract}
RESUMO: Croton cajucara Beth (Euphorbiaceae) uma espécie medicinal nativa da região Amazônica do Brasil, onde é vulgarmente conhecida como 'sacaca', representa um recurso terapêutico eficaz no tratamento e cura de várias doenças. O metabólito majoritário transdesidrocrotonina (DCTN), isolado das cascas do caule desta planta, encontra-se correlacionado com grande parte das propriedades medicinais da sacaca. Este artigo de revisão, descreve os resultados fitoquímicos e farmacológicos que foram realizados com o diterpeno do tipo clerodano DCTN, bem como seus derivados semi-sintéticos. Adicionalmente, apresenta perspectivas para a biodisponibilização deste protótipo de fármaco em nanosistemas.
\end{abstract}

Unitermos: Croton cajucara, trans-desidrocrotonina, DCTN, fitoquímica, atividade biológica, nanobiotecnologia.

\begin{abstract}
A review of the biologic activities of trans-dehydrocrotonin, a natural product obtained from Croton cajucara”. Croton cajucara Beth (Euphorbiaceae) is a plant found in the Amazonian Region of North Brazil, where it is popularly known as sacaca. The major secondary metabolite, trans-dehydrocrotonin (DCTN) a clerodane-type diterpene, isolated from the stem bark is a chief bioactive compound of Croton cajucara. This review describes results of extensive pharmacological studies of DCTN, as well as its semi-synthetic derivatives, and also presents insights into the use of DCTN as a therapeutic agent and some potential advantages of its incorporation in drug delivery systems.
\end{abstract}

Keywords: Croton cajucara, trans-dehydrocrotonin, phytochemistry, biologic activities, nanobiotechnology.

\section{INTRODUÇÃO}

Plantas têm sido tradicionalmente utilizadas por populações em todos os continentes no controle de diversas doenças e pragas, sendo reconhecidas mais de 13.000 espécies que são mundialmente consumidas como fármacos ou fonte de fármacos (Simões et al., 2004).

Dentre as famílias de plantas medicinais destaca-se a Euphorbiaceae, que possui mais de 8.000 espécies amplamente distribuídas em regiões tropicais e temperadas de todo o mundo. Apesar desta família ser amplamente distribuída na região Amazônica, algumas de suas espécies nunca foram estudadas do ponto de vista químico e muitas outras não foram classificadas. O gênero Croton, de acordo com Pax e Hoffman (1931), pertence à subfamília Crotonoideae, sendo uma das mais numerosas Euphorbiaceae. No Brasil há mais de 300 das cerca de 700 espécies desse gênero, a maioria delas com propriedades químicas e/ou farmacológicas conhecidas.
O gênero Croton tem representantes tanto medicinais, quanto tóxicos; uma das espécies mais conhecidas no Norte do Brasil é o Croton cajucara, vulgarmente conhecido por sacaca (termo correspondente a feitiço, na língua Tupi) (Abreu et al., 2001).

No estado do Pará, as folhas e cascas do caule do Croton cajucara são utilizadas na forma de chá ou pílula, no combate a diabetes, diarréia, malária, febre, problemas estomacais, inflamação do fígado, rins, vesícula e no controle de índices elevados de colesterol. A sacaca também é comercializada em farmácias de manipulação e, neste caso, o pó das cascas do caule é vendido em cápsulas (concentração aproximada de 250 $\mathrm{mg}$ ). As folhas são comercializadas em feiras livres da cidade de Belém-PA, para distúrbios do fígado e auxilia na digestão, principalmente após ingestão de alimentação rica em gorduras. O pó das folhas é vendido com indicação hepatoprotetora, para tratamento do diabetes e em dietas de emagrecimento (Maciel et al., 1998a; Maciel et al., 
2002a; Veiga Jr. et al., 2005).

Estudos fitoquímicos foram realizados com grandes quantidades de folhas e cascas do caule (partes utilizadas na medicina popular) de árvores variando entre 11/2-6 anos de idade, buscando o isolamento de substâncias majoritárias que pudessem vir a ter representatividade em testes biológicos. Estes estudos mostraram que as cascas do caule são ricas em diterpenos do tipo clerodano, tendo sido isolados: trans-desidrocrotonina (DCTN), transcrotonina (CTN), cis-cajucarina B, trans-cajucarina B, cajucarina A, cajucarinolida, isocajucarinolida, isosacacarina e o triterpeno ácido acetilaleuritólico (AAA) (Maciel et al., 1998b; Maciel et al., 2003).

Dentre os terpenóides isolados, a DCTN (Figura 1) foi o componente majoritário isolado (Maciel et al., 1998a,b) com um rendimento incomum (em se tratando de produto natural) de 1,4\%. Este 19nor-clerodano mostrou correlação com grande parte das propriedades farmacológicas do Croton cajucara, tendo sido comprovado as seguintes atividades: hipoglicêmica (Farias et al., 1997; Silva et al., 2001a), hipolipidêmica (Silva et al., 2001a,b,c; Bighetti et al., 2004), antigenotóxica (Agner et al., 1999; Agner et al., 2001), antiulcerogênica (Rodríguez et al., 2004; SouzaBrito et al., 1998; Hiruma-Lima et al., 1999; Melo et al., 2003; Maciel et al., 2000), antitumoral (Maciel et al., 2000; Grynberg et al., 1999; Melo et al., 2004), antiinflamatória e antinociceptiva (Maciel et al., 2002a; Carvalho et al., 1996), antiestrogênica (Luna Costa et al., 1999) e cardiovascular (Silva et al., 2005). Atualmente, a partir de modificações químicas da molécula de DCTN estão sendo desenvolvidos estudos que objetivam a avaliação da relação estrutura/atividade biológica (Melo et al., 2003; Maciel et al., 2000; Grynberg et al., 1999; Melo et al., 2004; Anazetti et al., 2003; Anazetti et al., 2004; Melo et al., 2001).

Este artigo apresenta uma revisão das diversas atividades biológicas da DCTN, destacando o seu potencial farmacológico no tratamento e/ou cura de várias doenças, bem como de seus derivados semi-sintéticos. Focaliza ainda, algumas vantagens do desenvolvimento de formulações farmacêuticas na liberação controlada de substâncias naturais, que poderão vir a ser utilizadas como fármacos.

\section{Estudos farmacológicos da DCTN}

\section{Atividade hipoglicêmica}

O tratamento com DCTN promoveu

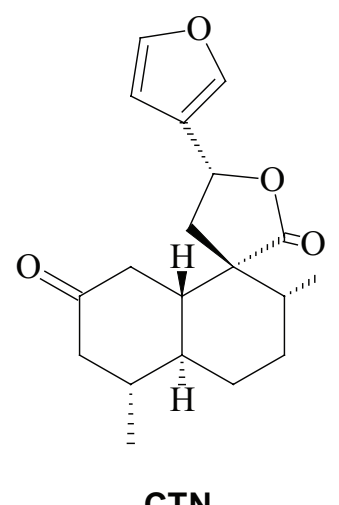

DCTN<smiles>CC1=CC(=O)C[C@H]2[C@@H]1CC[C@@H]1C[C@]23C(=O)O[C@@H](C2=C[C@H](O)OC2=O)C13</smiles>

CJCR<smiles>CC1=CC(=O)C[C@H]2[C@@H]1CC[C@@H](O)[C@@H]2C[C@@H](OC=O)C1=CC(=O)O[C@@H]1O</smiles>

ICJCR

Figura 1. Clerodanos isolados de Croton cajucara. 
hipoglicemia em ratos Wistar machos portadores do diabetes induzido por aloxano (Farias et al., 1997). Doses diferentes de DCTN (25 e $50 \mathrm{mg} / \mathrm{kg}$ ) foram administradas por via oral uma hora antes (tratamento preventivo), $24 \mathrm{e}$ 48 h depois da administração de aloxano (150 mg/kg, s.c.; fase estabelecida do diabetes), por três dias consecutivos. A glibenclamida, um fármaco recomendado atualmente na terapia do diabetes, foi utilizado como referência (2 $\mathrm{mg} / \mathrm{kg}$, v.o.). O efeito hipoglicemiante da DCTN foi evidenciado em ambas as doses, com redução dos níveis de glicose mais acentuada na concentração de $50 \mathrm{mg} / \mathrm{kg}$. A resposta foi tempo-dependente com diminuição de 36, 50 e $53 \%$, nos tempos de 1, 3 e 5 h após o tratamento, respectivamente. A DCTN também reduziu os níveis sangüíneos de glicose em animais hiperglicêmicos, sem provocar hipoglicemia como observado com o tratamento com a glibenclamida. Este fato constitui, portanto, uma vantagem terapêutica no controle do diabetes, visto que a hipoglicemia provocada pela glibenclamida em pacientes diabéticos é um fator de risco (Farias et al., 1997).

A ação hipoglicemiante da DCTN foi também avaliada em modelo de diabetes experimental induzida em ratos Wistar machos por uma única injeção intraperitoneal de estreptozotocina (STZ) na dose de $60 \mathrm{mg} / \mathrm{kg}$ (Silva et al., 2001a). Na primeira fase do experimento, realizou-se um pré-tratamento com DCTN (50 mg/kg v.o.) $1 \mathrm{~h}$ antes da indução com STZ, tendo sido utilizado a aminoguanidina (50 mg/kg, i.p.) como fármaco de referência. Na segunda fase do experimento, o efeito terapêutico da DCTN (50 mg/kg, v.o.) foi verificado na fase do diabetes estabelecido (48 h após indução com STZ). A DCTN demonstrou maior eficácia no regime de pré-tratamento com redução significativa da hiperglicemia de 58, 56 e 61\% após 24, 48 e 72 h, respectivamente. O efeito hipoglicemiante da DCTN foi semelhante àquele apresentado pela aminoguanidina, fármaco conhecido como inibidor da óxido nítrico sintase com propriedades antioxidante e antiradicais livres. $\mathrm{O}$ tratamento dos animais com a DCTN, na fase do diabetes estabelecido, promoveu uma redução significativa, porém transitória, da hiperglicemia 3 h após a administração do fármaco.

\section{Atividade hipolipidêmica}

O efeito hipolipidêmico da DCTN na redução dos níveis séricos de triglicerídeos e colesterol (Silva et al., 2001a) foi avaliado em ratos Wistar após indução de hipertrigliceremia com etanol a 26\% (1 mL/kg). A DCTN na dose de $50 \mathrm{mg} / \mathrm{kg}$ (v.o.) promoveu uma diminuição de $37 \%$ na taxa de triglicerídeos, além de reduzir significativamente os níveis de colesterol. Esse efeito hipogliceridêmico apresenta uma grande vantagem no tratamento de pacientes diabéticos não-insulina dependentes (diabetes tipo II), cujo metabolismo lipídico encontra-se alterado com aumento na mobilização de triglicerídeos e liberação de ácidos graxos não- esterificados do tecido adiposo para a corrente sanguínea (Melo et al., 2004).

Outro estudo avaliou a atividade da DCTN após indução da hipercolesterolemia e hipertriglicemia por Triton WR 1339 (tyloxapol) (400 mg/kg, i.p.) em ratos. Após tratamento oral com DCTN (25 e $50 \mathrm{mg} /$ $\mathrm{kg}$ ) e gemfibrozil (100 mg/kg) observou-se, após 24 h, uma redução significativa do nível total de colesterol em aproximadamente 40 e 52\%, respectivamente. Após 48 h, a redução correspondente foi de 39 e 28\%, respectivamente. No entanto, o gemfibrozil (100 mg/ $\mathrm{kg}$ ) causou diminuição de 35\% em 24 h e $46 \%$ em 48 h. Além disso, constatou-se uma atividade mais efetiva do gemfibrozil na redução dos níveis de triglicerídeos (Silva et al., 2001b). Este efeito também foi investigado em ratos alimentados com dieta de gordura por um período de duas semanas. A DCTN (após 4 h da alimentação) foi administrada oralmente uma vez ao dia, nas doses de 25 e $50 \mathrm{mg} / \mathrm{kg}$. Este fármaco diminuiu o colesterol total e os níveis de triglicerídeos em 20 e 30\% na dose $25 \mathrm{mg} / \mathrm{kg}$, e houve diminuição de 50 e $51 \%$ na dose $50 \mathrm{mg} / \mathrm{kg}$. Ao término do experimento, um aumento de $30 \%$ no peso dos animais foi observado para o grupo não tratado com DCTN (Silva et al., 2001c).

Recentemente, avaliou-se a atividade hipolipidêmica da DCTN utilizando ratos geneticamente modificados. Estes modelos simularam dois tipos de hiperlipidemia da genética humana. O tratamento oral com DCTN (100 mg/kg) durante 25 dias não apresentou resultados significativos na redução de lipídeos séricos. Embora vários estudos comprovem a atividade hipolipidêmica da DCTN (Silva et al., 2001a,b,c; Bighetti et al., 2004), o efeito hipolipidêmico desta droga pode estar relacionado às causas de hiperlipidemia não genética (Bighetti et al., 2004).

\section{Atividade antigenotóxica}

Fatores naturais e artificiais podem estar envolvidos com o aparecimento de mutações e desenvolvimento de câncer. A antigenotoxicidade da DCTN foi comprovada em células da medula óssea de camundongos Swiss in vivo, submetidos a um tratamento agudo pela via intraperitoneal, pelos seguintes parâmetros: micronúcleos, aberrações cromossômicas e índice mitótico, nas doses equivalentes a 25, 50 e 75\% da $\mathrm{DL}_{50}$ (Agner et al., 1999), previamente determinada em camundongos (555 mg/kg, v.o.) (Carvalho et al., 1996).

Em outro estudo, a ação antigenotóxica da DCTN foi confirmada nas mesmas doses e parâmetros do estudo anterior, avaliando a diferença relacionada às duas vias de administração: oral e intraperitoneal. A ciclofosfamida (10 mg/kg, i.p.) foi utilizada como controle positivo devido a sua forte ação clastogênica. Os resultados mostraram antimutagenicidade nas doses de 50 e $75 \%$ da $\mathrm{DL}_{50}$, quando comparados aos da ciclofosfamida. No entanto, a dose de $25 \%$ da $\mathrm{DL}_{50}$ só 
foi efetiva pelos métodos de micronúcleos e aberrações cromossômicas na administração oral. Baseado nestas observações pôde-se concluir que a via oral é a mais adequada para administração da DCTN. Isto é relevante, pois esta é a via de uso popular do Croton cajucara (Agner et al., 2001).

\section{Atividade antiulcerogênica}

A atividade antiulcerogênica da DCTN foi avaliada em quatro modelos de indução de lesões gástricas (úlcera de Shay, restrição hipotérmica, indução por etanol e indução por indometacina) em ratos. A dose aguda de DCTN foi de $100 \mathrm{mg} / \mathrm{kg}$, por via oral, em todos os modelos. No modelo da úlcera de Shay, a DCTN apresentou importante atividade anti-secretora na proteção da mucosa gástrica. No ensaio de restrição hipotérmica, a DCTN protegeu efetivamente a mucosa (semelhante a cimetidina) (Rodríguez et al., 2004; Souza-Brito et al., 1998; Maciel et al., 2000). Do mesmo modo, apresentou atividade gastroprotetora contra o efeito agressivo do etanol. Porém, no ensaio de indução de úlcera pela indometacina, a DCTN não produziu uma redução significante ao dano causado por este inibidor da síntese de prostaglandinas, tendo sido constatado que o efeito antiulcerogênico não está relacionado com a síntese ou retenção de muco, e sim por um possível aumento na produção de prostaglandinas (Rodríguez et al., 2004).

Estudos posteriores, envolvendo os mecanismos de ação da atividade antiulcerogênica da DCTN, comprovaram que este produto natural tem um excelente efeito preventivo em úlceras gástricas: suprime eficazmente a secreção ácida, age como antagonista não competitivo dos receptores envolvidos na secreção ácida do estômago e protege a mucosa gástrica por um aumento de prostaglandinas $\left(\mathrm{PGE}_{2}\right.$ ) (Hiruma-Lima et al., 1999; Melo et al., 2003).

A atividade antiulcerogênica foi também comprovada, para o 19-nor-clerodano trans-crotonina, que difere da DCTN apenas pela ausência da insaturação $\Delta^{3,4}$ do anel A do sistema decalínico, bem como para o óleo fixo das cascas do caule do Croton cajucara (Almeida et al., 2003; Hiruma-Lima et al., 2000; Hiruma-Lima et al., 2002). Como os clerodados CTN, cis-cajuarina B e transcajuarina B foram evidenciados em baixos percentuais, neste óleo, (Souza et al., 2006) deve existir uma ação conjunta destes clerodanos para o efeito gastroprotetor deste óleo.

\section{Atividades antinflamatória e antinociceptiva}

O efeito antiinflamatório da DCTN foi avaliado utilizando-se os métodos de edema de pata (induzido por carragenina) e granuloma (causado por pellet de algodão em ratos) e o efeito antinociceptivo, por indução de contrações (com ácido acético) e por teste de termossensibilidade.
A indução do edema de pata pela carragenina (em ratos) foi realizada trinta minutos após a administração intraperitoneal de DCTN (50 mg/kg); 0,1 mL (100 $\mu \mathrm{g})$ de carragenina foi injetado na pata esquerda e $0,1 \mathrm{~mL}$ de solução salina na pata direita. Os resultados foram obtidos medindo-se a diferença de volume de ambas as patas. A indometacina (10 mg/kg, v.o.) foi utilizada como fármaco de referência para atividade antiinflamatória. A administração da DCTN antes da indução da inflamação, inibiu a formação de edema em 38, 35 e 38\% na primeira, segunda, e terceira hora, respectivamente. Aindometacina, na terceira hora, inibiu o edema em $43 \%$. No granuloma, 4 pellets (pesando $160 \mathrm{mg}$ ) foram esterilizados e implantados subcutaneamente no abdômen dos animais em quatro posições simétricas. Durante 6 dias, os diferentes grupos de animais foram tratados oralmente com 0,5 mL de veículo (grupo controle), dexametasona $0,5 \mathrm{mg} / \mathrm{kg}$, DCTN 50 e $200 \mathrm{mg} / \mathrm{kg}$. No $7^{\circ} \mathrm{dia}$, os animais foram sacrificados e os tecidos granulomatosos foram removidos. Os pellets foram secados a $60{ }^{\circ} \mathrm{C}$ e pesados. A administração diária de 50 e $200 \mathrm{mg} / \mathrm{kg}$ de DCTN, neste modelo, mostrou no sétimo dia, uma inibição da inflamação em 11 e 49\%, respectivamente (Carvalho et al., 1996).

Para a avaliação da atividade antinociceptiva da DCTN na indução de contrações com ácido acético, os grupos de animais (ratos) foram tratados oralmente com DCTN nas doses de 10, 20, 50 e $100 \mathrm{mg} / \mathrm{kg}, 30 \mathrm{~min}$ antes da injeção intraperitoneal da solução de ácido acético 0,6\% (0,25 mL/kg). O número de contrações musculares foi contado durante $20 \mathrm{~min}$, iniciando-se no $5^{\circ}$ minuto após a indução da dor. A administração oral de DCTN inibiu o número de contrações musculares de maneira dose-dependente. A $\mathrm{DE}_{50}$ obtida para este parâmetro foi $52,08 \mathrm{mg} / \mathrm{kg}$. No teste de termossensibilidade, os animais foram colocados em uma chapa de metal mantida a 50 $\pm 0,5^{\circ} \mathrm{C}$. O tempo de reação foi observado pela ação dos animais que lambem as patas traseiras e/ou fazem movimentos saltitantes, antes e depois da administração da DCTN. O período de exposição foi de 20 segundos. Neste experimento, a morfina 4,0 mg/kg, i.p. foi utilizada como droga de referência e comprovou-se que a DCTN (50 mg/kg, v.o.) não apresentou resposta significativa (Carvalho et al., 1996).

Posteriormente, os efeitos antinociceptivo e antiinflamatório da DCTN foram reavaliados em doses mais elevadas, tendo sido confirmados (Maciel et al., 2002a). Para a avaliação da antinocicepção, foi utilizado ácido acético a $1,2 \%(0,1 \mathrm{~mL} / 10$ g, i.p.) e DCTN (150 e $300 \mathrm{mg} / \mathrm{kg}$, v.o.). A DCTN na dose mais elevada (300 mg/kg) reduziu em 31\% o número de contrações abdominais. Na avaliação da atividade antiinflamatória, a DCTN (100 e $300 \mathrm{mg} / \mathrm{kg}$, v.o.) foi administrada 60 min antes da indução de lesão por carragenina (1\% em solução salina; $250 \mu \mathrm{L} /$ animal). A inibição da migração de leucócitos para o líquido pleural pela DCTN foi de 33\%, enquanto a dexametasona (fármaco padrão) apresentou 
inibição de 44\% (Maciel et al., 2002a).

\section{Atividade antiestrogênica}

Com objetivo de avaliar o efeito antiestrogênico da DCTN foram utilizadas ratas imaturas (19-21 dias) pelo modelo de bioensaio para estrógeno. Os antiestrogênicos inibem a cornificação vaginal e o aumento uterino. Nestes testes foram utilizados três grupos de animais: o primeiro grupo recebeu o veículo (óleo de milho, 5 $\mathrm{mL} / \mathrm{kg}$, s.c. + DMSO a $3 \%, 10 \mathrm{~mL} / \mathrm{kg}$, v.o.); o segundo grupo recebeu apenas estrógeno (10 mg/animal, s.c.) e o terceiro, estrógeno e DCTN (25 e $50 \mathrm{mg} / \mathrm{kg}$, v.o., respectivamente) durante 3 dias sucessivos. No quarto dia, todos os animais foram observados quanto à abertura vaginal e posteriormente sacrificados. Os pesos uterinos relativos foram obtidos e expressados em miligramas por $30 \mathrm{~g}$ de peso corporal. O tratamento com estrógeno aumentou cinco vezes o peso uterino e abertura vaginal comprovada em $100 \%$ dos animais. No entanto, o grupo de animal que recebeu DCTN + estrógeno apresentou redução significativa do peso uterino, além de inibição da abertura vaginal em $100 \%$ dos animais. A DCTN sozinha não produziu nenhuma mudança. Neste estudo não há precisão em relação ao mecanismo de ação da DCTN na redução do crescimento uterino estrógeno-induzido. A DCTN diminuiu o peso uterino, como também inibiu a abertura vaginal induzida através do estrógeno em ratos imaturos; desta forma é concebível que a DCTN seja antiestrogênica (Luna Costa et al., 1999).

\section{Atividade cardiovascular}

Estudos in vivo e in vitro revelaram a atividade cardiovascular da DCTN. Em ratos Wistar machos, a DCTN (10 e 15 mg/kg, i.v.) causou redução na pressão sangüínea e freqüência cardíaca. Observou-se um efeito vasorrelaxante (em experimentos in vitro) no seguimento da artéria aorta isolada de ratos, e no átrio direito também isolado de ratos, observou-se indução de um efeito cronotrópico negativo. Os efeitos hipotensivo e bradicardíaco da DCTN foram possivelmente correlacionados com a liberação de óxido nítrico e com os efeitos diretos causados na musculatura lisa vascular e na atividade do ritmo cardíaco (Silva et al., 2005).

Citotoxicidade e atividade antitumoral: relação estrutura-atividade da DCTN e seus derivados semisintéticos

A atividade citotóxica da DCTN foi estudada em diversas células tumorais das seguintes linhagens: células fibroblásticas pulmonares de hamsters chineses (Souza-Brito et al., 1998; Grynberg et al., 1999; Melo et al., 2001; Rodriguez; Haun, 1999; Freire et al., 2003), células promielocíticas leucêmicas (HL60) (Anazetti et al., 2003; Anazetti et al., 2004; Corrêa et al., 2005), hepatócitos de ratos (Rodriguez; Haun, 1999; Corrêa et

al., 2005) e células de carcinoma de Ehrlich (Maciel et al., 2002a; Grynberg et al., 1999; Melo et al., 2004).

O efeito citotóxico da DCTN verificado em células fibrobláticas pulmonares de hamsters chineses (V79) nas concentrações variando de 80 a $400 \mu \mathrm{M}$, mostrou uma redução dose-dependente da viabilidade celular com $\mathrm{CI}_{50}$ de $240 \mu \mathrm{M}$ (Souza-Brito et al., 1998). Adicionalmente, a atividade citotóxica da DCTN complexada a $\beta$-ciclodextrina foi testada com o objetivo de controlar a liberação da DCTN em células V79 e cultura de hepatócitos, tendo sido observado uma diminuição da citotoxicidade da DCTN complexada (Freire et al., 2003). O estudo em células promielocíticas leucêmicas (HL60) e células mononucleares sanguíneas humanas, mostrou diferenciação celular e indução de apoptose em células do tipo HL60 e baixa toxicidade nas células sanguíneas (Anazetti et al., 2003; Anazetti et al., 2004). A citotoxicidade da DCTN $(8 \mu \mathrm{M})$, analisada em hepatócitos extraído de ratos, comprovou pelos danos causados nas células hepáticas após tratamento subcrônico da DCTN, a ocorrência de toxicidade seletiva (Rodriguez; Haun, 1999). Outro estudo mostrou a influência da DCTN na inibição de fosfatase em células HL60, induzindo apoptose (Corrêa et al., 2005).

Em decorrência da importância farmacológica da DCTN, bem como do seu elevado teor de isolamento (Maciel et al., 1998a; Maciel et al., 2000), vários pesquisadores reportaram a obtenção de derivados deste clerodano, tais como: a CTN-derivado (obtida da DCTN por hidrogenação catalítica) (Maciel et al., 2002a; Maciel et al., 2000; Grynberg et al., 1999), os butenolídeos cajucarinolida (CJCR) e isocajucarinolida (ICJCR) (obtidos pela fotooxidação da subunidade furânica) (Maciel et al., 2002a; Maciel et al., 2007), os álcoois derivados DCTN-OL (DCTN reduzida com $\mathrm{NaBH}_{4}$, na posição-2) e DCTN-tri-OL (DCTN reduzida com $\mathrm{LiAlH}_{4}$, na posição-2 e com abertura da subunidade lactona) e a amida derivado DCTN-2-ona-20-amida (Melo et al., 2003; Anazetti et al., 2003; Anazetti et al., 2004; Melo et al., 2004) (Figura 2). Dentre estes derivados, a CTN e os clerodanos-butenolídeos CJCR e ICJCR se destacam por serem de ocorrência natural, tendo sido isolados como constituintes minoritários das cascas do caule do Croton cajucara (Maciel et al., 1998a,b). Desta forma, a semi-síntese destes componentes permitiu a realização de testes biológicos; a CTN-derivado por exemplo, provou ser um agente antiulcerogênico eficaz (Maciel et al., 2000; Melo et al., 2004; Hiruma-Lima et al., 2002). No entanto, a perda da insaturação $\Delta^{3,4}$ (CTN-natural ou derivado, por comparação com a DCTN) reduziu a atividade antitumoral da molécula em testes in vivo em tumores ascíticos, e in vitro em linhagens de células tumorais ascíticas (Grynberg et al., 1999). Nestes experimentos, a citotoxicidade de ambos DCTN e CTN-derivado foi avaliada em células de carcinoma de Ehrlich utilizando o método do MTT para avaliar a viabilidade celular após contato de 24 a $48 \mathrm{~h}$. Os valores de $\mathrm{CI}_{50}$ foram de 52,2 
$\mu \mathrm{g} / \mathrm{mL}(16 \mu \mathrm{M})$ e $51,8 \mu \mathrm{g} / \mathrm{mL}(16 \mu \mathrm{M})$ para DCTN e CTN-derivado, respectivamente. Nenhuma indução da fragmentação nucleossomal do DNA foi observada após tratamento com DCTN ou CTN-derivado $(50 \mu \mathrm{g} / \mathrm{mL})$ (Grynberg et al., 1999).

Nos testes que avaliaram a atividade antitumoral da DCTN e da CTN-derivado, foram utilizados camundongos DBA/2 (fêmeas) em dois modelos experimentais de tumores ascíticos: sarcoma 180 (S180) e carcinoma de Ehrlich (CE). A DCTN apresentou atividade contra S-180 e CE nas doses de $84 \mathrm{mg} / \mathrm{kg}$ (2 dias consecutivos) e $120 \mathrm{mg} / \mathrm{kg}$ (3 dias consecutivos) após administração intraperitoneal, respectivamente (Grynberg et al., 1999). Este efeito foi comparável à ação da 5-fluoruracila, um potente anticancerígeno, em S-180 (38 mg/kg; 2 dias consecutivos) e CE (80 mg/ $\mathrm{kg}, 3$ dias consecutivos). No entanto, a CTN-derivado, não apresentou atividade antitumoral nas mesmas doses testadas. Adicionalmente, foi verificado um aumento significativo $(\mathrm{p}<0.05)$ na indução do fator de necrose tumoral ( $\alpha$-TNF) após tratamento dos animais com DCTN na dose de $120 \mathrm{mg} / \mathrm{kg}$. Este resultado sugere que o tratamento com a DCTN prolonga a sobrevida dos animais com S-180 ou CE devido a uma ação direta sobre o tumor do $\alpha$-TNF secretado na área de crescimento celular do tumor, de forma semelhante àquela do taxol (Mullins et al., 1997). A citotoxicidade dos clerodanos-butenolídeos CJCR e ICJCR foi avaliada em células de carcinoma de Ehrlich e os valores de $\mathrm{IC}_{50}$ foram de $22,4 \mu \mathrm{g} / \mathrm{mL}$ (65 $\mu \mathrm{M})$ e $3,5 \mu \mathrm{g} / \mathrm{mL}(10 \mu \mathrm{M})$, respectivamente (Maciel et al., 2002a; Maciel et al., 2007). A Tabela 1 mostra resumidamente os resultados citotóxicos e atividade antitumoral da DCTN e seus derivados semi-sintéticos em células de carcinoma de Ehrlich e em camundongos com sarcoma 180 ou carcinoma de Ehrlich.

Os derivados semi-sintéticos acima mencionados foram avaliados em diferentes estudos para a determinação da citotoxicidade e atividade antitumoral, na tentativa de melhor entender a relação entre a estrutura química e a atividade biológica da DCTN, bem como de elucidar o mecanismo de ação deste clerodano. A obtenção de derivados da DCTN possibilita ainda, a aquisição de novas substâncias biologicamente eficazes que poderão vir a ser isoladas de fontes naturais, a exemplo da CTN, CJCR e ICJCR.

Em outro estudo de citotoxicidade com células de carcinoma de Ehrlich, com viabilidade celular avaliada pelo método do MTT, após 72 h de incubação, a DCTN e seus álcoois derivados DCTN-OL e DCTNtri-OL não apresentaram citotoxicidade. No entanto, o derivado DCTN-2-ona-amida foi eficaz $\left(\mathrm{CI}_{50}\right.$ de $400 \mu \mathrm{M}$ e inibição de $20 \%$ da atividade fosfatase) (Melo et al., 2004). Neste experimento, foram utilizados camundongos machos BALB/c com tumor ascítico de Ehrlich, que foram tratados com doses de 2,5 a $20 \mathrm{mg} / \mathrm{kg}$ de peso. O tratamento com a molécula-mãe DCTN, aumentou a sobrevida dos animais de 25 para 35 dias, enquanto que seus derivados (DCTN-OL, DCTN-tri-OL e DCTN2-ona-20-amida) não foram eficazes. Um resultado extraordinário da cura de $80 \%$ dos animais ( $<<0,001$ ), foi obtido após tratamento com a DCTN na dose de 80 $\mathrm{mg} / \mathrm{kg}$ (Melo et al., 2004).

O efeito antitumoral mediado por células natural killer (NK) foi avaliado para melhor compreensão do mecanismo de ação deste clerodano (DCTN), bem como para uma possível confirmação da ação imunomoduladora da DCTN. Os resultados demonstraram que a DCTN na dose de $20 \mathrm{mg} / \mathrm{kg}$ induziu de forma significativa ( $\mathrm{p}<$ 0,05 ) a atividade esplênica de células NK, apesar de não ser observada esplenomegalia nos animais (Melo et al., 2004). A hipótese da ação imunomoduladora (Grynberg et al., 1999) fica, portanto, fortalecida, visto que a DCTN age de forma parcialmente indireta na redução do tumor, por modulação da função imune dos animais (Melo et al., 2004).

A atividade antiulcerogênica dos derivados DCTN-OL, DCTN-tri-OL e DCTN-2-ona-20-amida, também foi avaliada. Apenas o derivado DCTN-OL se mostrou ativo em testes in vivo, sugerindo desta forma, que a presença de ambos carbonila $\alpha, \beta$-insaturada (insaturação $\Delta^{3,4}$ ) e subunidade lactona, são essenciais para a atividade antiulcerogênica de compostos terpenoídicos (Melo et al., 2003). Esta sugestão está de acordo com Wattenberg que postulou que compostos contendo em sua estrutura uma carbonila $\alpha, \beta$-insaturada apresentam uma maior interação com receptores que induzem o metabolismo de agentes xenobióticos, devido à sua ação em enzimas do organismo (Wattenberg, 1995). No entanto, ambos os clerodanos DCTN [carbonila (C2) $\alpha, \beta$-insaturada $\left.\left(\Delta^{3,4}\right)\right]$ e CTN (carbonila-C2) apresentaram efeito antiulcerogênico. Neste caso, é provável que o anel A do sistema decalínico destes clerodanos, não esteja diretamente envolvido na sua atividade antiulcerogênica, estando portanto, a subunidade lactona (Maciel et al., 2000; Hiruma-Lima et al., 2002).

\section{Mecanismo de ação da DCTN}

Certos grupos funcionais são essenciais para a atividade biológica de terpenóides, como os aceptores de Michael (cetona $\alpha, \beta$-insaturada e $\alpha$-metileno- $\gamma$ lactona) que são reconhecidos como grupamentos químicos responsáveis pelas atividades antiulcerogênica (Giordano et al., 1992) e antitumoral (Nakayachi et al., 2004; Ahn; Sok, 1996) de compostos orgânicos em geral. Como exemplo específico podemos citar os resultados citotóxicos observados na relação estrutura/ atividade de lactonas sesquiterpênicas, onde três grupos químicos presentes nas moléculas desses compostos contribuem para a atividade biológica (Mullins et al., 1997; Wattenberg, 1995), dentre eles, destaca-se o grupo $\alpha$-metileno- $\gamma$-lactona, que participa de reações do tipo Michael com moléculas biológicas e inativa enzimaschaves (Kupchan et al., 1971). Esse grupo é essencial para 
<smiles>CC1=C[C@H](O)C[C@H]2[C@@H]1CC[C@@H](C)[C@]21C[C@H](c2ccoc2)OC1=O</smiles>

DCTN-OL<smiles>CC1=C[C@@H](O)C[C@H]2[C@@H]1CC[C@H](C)[C@]2(CO)C[C@H](O)c1ccoc1</smiles>

DCTN-tri-OL<smiles>CC1=CC(=O)C[C@H]2[C@@H]1CC[C@H](C[C@H](O)c1ccoc1)[C@H]2C(=O)N(C)C</smiles>

DCTN-2-ona-20-amida

Figura 2. Derivados semi-sintéticos obtidos da DCTN.

Tabela 1. Citotoxicidade e atividade antitumoral da DCTN e seus derivados semi-sintéticos em células de carcinoma de Ehrlich e em camundongos com sarcoma 180 (S180) ou carcinoma de Ehrlich (CE).

\begin{tabular}{l|l|l|l}
\hline Clerodanos & $\mathrm{CI}_{50}(\mu \mathrm{g} / \mathrm{mL})$ & $\begin{array}{l}\text { Atividade antitumoral T/C } \\
\%\end{array}$ & Referências \\
\hline $\begin{array}{l}\text { trans-desidrocrotonina } \\
\begin{array}{l}\text { DCTN) } \\
\text { (substância natural) }\end{array}\end{array}$ & $52,2(16 \mu \mathrm{M})$ & $\begin{array}{l}137(\mathrm{~S} 180 ; 84 \mathrm{mg} / \mathrm{kg}) \\
128(\mathrm{CE} ; 120 \mathrm{mg} / \mathrm{kg}) \\
\text { aumento de } 80 \% \\
\text { de sobrevida } \\
(\mathrm{CE} ; 20 \mathrm{mg} / \mathrm{kg})\end{array}$ & Grynberg et al., 1999 \\
\hline $\begin{array}{l}\text { trans-crotonina (CTN) } \\
\text { (natural e semi-sintética) }\end{array}$ & $51,8(16 \mu \mathrm{M})$ & pouco eficaz & Melo et al., 2004 \\
\hline $\begin{array}{l}\text { cajucarinolida (CJCR) } \\
\text { (natural e semi-sintética) }\end{array}$ & $22,4(65 \mu \mathrm{M})$ & n.a. & Maciel et al., 2002a \\
\hline $\begin{array}{l}\text { iso-cajucarinolida (ICJCR) } \\
\text { (natural e semi-sintética) }\end{array}$ & $3,5(10 \mu \mathrm{M})$ & n.a. & Maciel et al., 2002a \\
\hline $\begin{array}{l}\text { DCTN-OL } \\
\text { (semi-sintética) }\end{array}$ & não citotóxico & inativo & Melo et al., 2004 \\
\hline $\begin{array}{l}\text { DCTN-tri-OL } \\
\text { (semi-sintética) }\end{array}$ & não citotóxico & inativo & Melo et al., 2004 \\
\hline $\begin{array}{l}\text { DCTN-2-OL-20-amida } \\
\text { (semi-sintética) }\end{array}$ & $400 \mu \mathrm{M}$ & inativo & Melo et al., 2004 \\
\hline
\end{tabular}

$\mathrm{T} / \mathrm{C} \%$ = [tempo médio de sobrevida dos animais tratados (dias)/(tempo médio de sobrevida dos animais controle (dias)] x 100 . $\mathrm{T} / \mathrm{C} \% \geq 125$, atividade antitumoral significativa; n.a. = não avaliado. 
a atividade antitumoral da DCTN, já que a abertura do anel lactona provocou a perda deste tipo de atividade da molécula (Melo et al., 2004). A subunidade ciclopentanona $\alpha, \beta$-insaturada da DCTN, também é essencial para a sua atividade antitumoral, já que o derivado CTN em doses similares, mostrou-se bem menos eficaz (Grynberg et al., 1999). Adicionalmente, a redução da carbonila $\alpha, \beta$ insaturada da DCTN se mostrou como uma alternativa inviável quando se desejou aumentar a atividade biológica do composto natural, pela obtenção dos álcoois derivados DCTN-OL e DCTN-tri-OL (Melo et al., 2004).

A spiro-lactona saturada presente na estrutura da DCTN não pode ser considerada como um genuíno aceptor de Michael, porém verifica-se que a abertura da lactona ocasiona diminuição das atividades antitumoral e antiulcerogênica, como verificado para estes álcoois-derivados. Com relação à intensa atividade antiulcerogênica da DCTN-2-ona-amida (lactona é aberta gerando um grupo amida), justifica-se considerando o grupo amida $\left[\mathrm{C}(\mathrm{O}) \mathrm{N}\left(\mathrm{CH}_{3}\right)_{2}\right]$ como um aceptor de Michael substituto (Anazetti et al., 2003; Anazetti et al., 2004).

Os resultados obtidos com a DCTN e seus derivados DCTN-2-ona-20-amida, CJCR e ICJCR permitem confirmar a hipótese sugerida por Grynberg et al. (1999) de que compostos contendo o grupo reativo carbonilas $\alpha, \beta$-insaturadas na sua estrutura são capazes de se ligarem a receptores celulares que induzem o aumento de atividade de enzimas de "fase II", responsáveis pela metabolização de compostos xenobióticos. Visto que estes clerodanos apresentam esta espécie química eletrofílica reativa, provavelmente ocorre interação química com biomoléculas que favorecem sua atividade antitumoral.

O mecanismo de ação da atividade antitumoral da DCTN continua em aberto, não tendo sido completamente elucidado. No entanto, avanços foram obtidos, tendo sido evidenciada apoptose (Corrêa et al., 2005) e uma possível atividade imunomoduladora pelo aumento de atividade de células NK após tratamento de animais com carcinoma de Ehrlich (Melo et al., 2004).

\section{Toxicidade e viabilização da biodisponibilização da DCTN}

A toxicidade subaguda da DCTN foi investigada em ratos Wistar adultos. Os animais (40 machos e 40 fêmeas) foram aleatoriamente distribuídos em 4 grupos (1 controle e 3 tratados). A DCTN foi administrada via oral na forma de suspensão em Tween 80 (12 \%) nas doses de 25, 50 e $100 \mathrm{mg} / \mathrm{kg}$ durante 35 dias. Após este período, observou-se um aumento significativo no peso do fígado de todos os animais que receberam doses mais elevadas. A redução nos níveis de fosfatase alcalina e colesterol, bem como um aumento na $\gamma$-glutamil transpeptidase (na dose $100 \mathrm{mg} / \mathrm{kg}$ ) foi observado em ratas. A DCTN causou alterações histopatológicas no fígado que incluiu tumefação turva, degeneração microvacuolar e alterações nucleares (Rodríguez et al., 2004).

A toxicidade aguda da DCTN foi observada por um período de $72 \mathrm{~h}$ após a administração oral. $\mathrm{A} \mathrm{DL}_{50}$ da DCTN foi de 555,0 mg/kg (v.o.) em camundongos, dez vezes mais alta do que a $\mathrm{DE}_{50}$ (Maciel et al., 2000). Neste estudo nenhum sintoma que justificasse a ação tóxica ao nível de sistema nervoso central, como estereotipia, ataxia e convulsão, foi detectado. A toxicidade aguda da DCTN foi também avaliada em camundongos Swiss machos. Após jejum de $12 \mathrm{~h}$, os animais receberam tratamento com a DCTN por via oral $(125,250,500,750$ e 1000 $\mathrm{mg} / \mathrm{kg}$ ) e intraperitoneal (31, 25, 62,5; 125, 250 e 500 $\mathrm{mg} / \mathrm{kg}$ ). O número de sobreviventes foi monitorado duas vezes ao dia, num período de 14 dias. Os valores de $\mathrm{DL}_{50}$ obtidos por via oral e intraperitoneal foram de $876 \mathrm{mg} / \mathrm{kg}$ (12 h) e de $47,2 \mathrm{mg} / \mathrm{kg}$, respectivamente. Os resultados obtidos mostraram a baixa toxicidade da DCTN (SouzaBrito et al., 1998).

Apesar dos estudos de toxicidade aguda e subcrônica da DCTN em animais mostrarem uma baixa toxicidade, o uso indevido da sacaca (folhas com indicação de cascas em tratamentos prolongados) na medicina alternativa pode acarretar hepatite tóxica (Maciel et al., 2006; Soares, 2004). Pelo menos em duas capitais (Belém e Rio de Janeiro), o uso dessa planta já mostrou que pode provocar efeitos hepatotóxicos irreversíveis (Maciel et al., 2002a,b; Veiga Jr. et al., 2005; Soares, 2004). Casos de hepatite aguda, crônica e fulminante foram notificados na região Amazônica e em outras regiões do país, em pacientes que fizeram uso da sacaca no controle de peso e redução dos níveis de colesterol (Maciel et al., 2002b; Maciel et al., 2006). O hospital Universitário da Universidade Federal do Pará possui um histórico significativo de pessoas vitimadas por doenças do fígado, as quais estavam ingerindo folhas em prolongadas dietas de emagrecimento. No entanto, o alerta sobre a toxicologia das folhas desta planta, em Belém, é um antigo conhecido da população, que, mesmo avisada, costuma consumir as folhas do Croton cajucara em dietas de emagrecimento; neste caso, os usuários se dizem encorajados pelo percentual de pessoas que garantem ter emagrecido com saúde (Maciel et al., 2002a,b; Maciel et al., 2006; Veiga Jr. et al., 2005).

Estudo recente realizado em pacientes da região Amazônica, que fizeram uso da sacaca durante 36 meses registrou 25 casos de hepatotoxicidade atribuídos à sacaca, tendo sido evidenciado que 21 pessoas tiveram hepatite aguda, 3 hepatite crônica e 1 hepatite fulminante (Soares, 2004).

A biodisponibilização da DCTN, bem como do Croton cajucara poderia ser otimizada via controle da liberação de fármacos com significante diminuição de efeitos tóxicos, através da redução da dose terapêutica. Neste contexto, a microencapsulação de fármacos permite: (a) o aumento de maneira racional e efetiva da eficácia terapêutica, bem como a diminuição da toxicidade dos fármacos pela redução dos picos de 
concentração plasmática máxima, objetivando manter em nível constante o fármaco no sangue; (b) o controle e prolongação da liberação do fármaco, reduzindo a freqüência de doses; (c) a otimização da atividade biológica, resultando em doses mais baixas devido a sítio-especificidade e a proteção da substância terapêutica de efeitos deletérios dos fluidos do organismo (Berkland et al., 2002). Desta forma, a nanotecnologia farmacêutica representa uma das fronteiras das ciências farmacêuticas que envolve aspectos multidisciplinares para desenvolvimento de sistemas nanométricos carreadores de fármacos, contribuindo para o melhoramento da biodisponibilidade de substância bioativas (Ravi-Kumar, 2000).

Diversos trabalhos comprovaram o aumento da eficácia e a redução da toxicidade de substâncias bioativas de origem natural ou sintética, administrados em lipossomas (Ratner, 2002), sistemas nanoparticulados (Feng et al., 2004; Berkland et al, 2002) ou micropartículas (Gupte; Ciftci, 2004; Ribeiro-Costa et al., 2004). Relatos recentes demonstram a atividade antitumoral do taxol (Gupte; Ciftci, 2004) e do ácido úsnico (Cladonia substellata) em microesferas (Ribeiro-Costa et al., 2004), da lectina de Cratylia mollis encapsulada em lipossomas (Andrade et al., 2004), de xantonas (Teixeira et al., 2005), da doxorrubicina em nanopartículas (Barraud et al., 2005) e 5-fluorouracil em lipossomas (Jin et al., 2005), atividade antiparasitária do praziquantel em lipossomas (Mourão et al., 2005), atividade antibiótica da vancomicina em micropartículas (Le Ray et al., 2005) e anfotericina B em lipossomas (Bellocchio et al., 2005), atividade hipoglicemiante da insulina em micropartículas (Kim et al., 2005) e anti-estrógenos em lipossomas e nanopartículas para tratamento do câncer de mama (Maillard et al., 2005; Memisoglu-Bilensoy et al., 2005).

\section{CONCLUSÃO}

Diversos estudos farmacológicos comprovam a eficácia biológica da DCTN, um diterpeno do tipo 19nor-clerodano isolado do Croton cajucara, uma espécie medicinal amplamente utilizada na região Amazônica do Brasil. Estes estudos instigam pesquisadores para a aquisição de dados científicos adicionais que sejam satisfatórios para a elucidação do seu mecanismo de ação. Em decorrência dos elevados teores de isolamento da DCTN e diante da possibilidade deste componente majoritário ser o responsável por efeitos hepatotóxicos que podem ser desencadeados em tratamentos prolongados (com o uso das cascas do caule do Croton cajucara), há a necessidade de se ampliar o desenvolvimento de novas alternativas terapêuticas que possam melhorar as atividades biológicas avaliadas em ensaios pré-clínicos e diminuir os possíveis efeitos adversos associados a este produto natural.

O mecanismo de ação antitumoral da DCTN continua não completamente elucidado. No entanto, avanços foram obtidos, tendo sido evidenciado a indução de apoptose e uma possível atividade imunomoduladora. Apesar dos estudos de toxicidade aguda e subcrônica da DCTN em animais mostrarem uma baixa toxicidade, o uso indevido da sacaca na medicina alternativa, pode acarretar efeitos hepatotóxicos irreversíveis. Portanto, uma das estratégias viáveis para permitir o uso terapêutico da DCTN consiste na incorporação deste diterpeno em sistemas de liberação controlada de fármacos, visando melhoria da biodisponibilidade e redução de possíveis efeitos hepatotóxicos que estejam envolvidos com este produto natural.

\section{AGRADECIMENTOS}

Os autores agradecem ao Conselho Nacional de Desenvolvimento Científico e Tecnológico-CNPq e a Rede Nanobiotec MCT/CNPq, bem como ao Programa PRODOC da CAPES.

\section{REFERÊNCIAS}

Abreu AS, Barbosa OS, Muller AH, Guilhon GMSP 2001. Revista Virtual de Iniciação Acadêmica da UFPA 1: 1. (http://www.ufpa.br/revistaic).

Agner AR, Maciel MAM, Pinto AC, Pamplona SGSR, Cólus IMS 1999. Investigation of genotoxic activity of trans-dehydrocrotonin, a clerodane diterpene from Croton cajucara. Teratogen Carcinogen Mutagen 19: 377-384.

Agner AR, Maciel MAM, Pinto AC, Cólus IMS 2001. Antigenotoxicity of trans-dehydrocrotonin, a clerodane diterpene from Croton cajucara. Planta Med 67: 815-819.

Ahn BZ, Sok DE 1996. Michael acceptors as a tool for anticancer drug design. Curr Pharm Design 2: 247262.

Almeida ABA, Miotto AM, Nunes DS, Spadari-Bratifisch RC, Souza-BritoARM 2002. Mechanism of antiulcerogenic activity of semi-synthetic crotonin obtained from Croton cajucara Benth. Rev Bras Farmacogn 12(Supl. 1): $105-110$.

Almeida ABA, Melo PS, Hiruma-Lima CA, Gracioso JS, Carli L, Nunes CS, Haun M, Souza-Brito ARM 2003. Antiulcerogenic effect and cytotoxic activity of semisynthetic crotonin obtained from Croton cajucara Benth. Eur J Pharmacol 472: 205-212.

Anazetti MC, Melo PS, Durán N, Haun M 2003. Comparative cytotoxicity of dimethylamide-crotonin in the promyelocytic leukemia cell line (HL60) and human peripheral blood mononuclear cells. Toxicology 188: 261-274.

Anazetti MC, Melo PS, Durán N, Haun M 2004. Dehydrocrotonin and its derivative, dimethylamide-crotonin induce apoptosis with lipid peroxidation and activation of caspases-2, -6 and -9 in human leukemic cells HL60. Toxicology 203: 123-137. 
Andrade CAS, Correia MTS, Coelho LCBB, Nascimento SC, Santos-Magalhães NS 2004. Antitumor activity of Cratylia mollis lectin encapsulated into liposomes. Int J Pharm 278: 435-445.

Barraud L, Merle P, Soma E, Lefrançois L, Guerret S, Chevallier M, Dubernet C, Couvreur P, Trepo C, Vitvitski L 2005. Increase of doxorubicin sensitivity by doxorubicinloading into nanoparticles for hepatocellular carcinoma cells in vitro and in vivo. J Hepatol 42: 736-743.

Bellocchio S, Gaziano R, Bozza S, Rossi G, Montagnoli C, Perruccio K, Calvitti M, Pitzurra L, Romani L 2005. Liposomal amphotericin B activates antifungal resistance with reduced toxicity by diverting Tolllike receptor signalling from TLR-2 to TLR-4. $J$ Antimicrob Chemother 55: 214-222.

Berkland C, King M, Cox A, Kim KK, Pack DW 2002. Precise control of PLG microsphere size provides enhanced control of drug release rate. $J$ Control Release 82: 137-147.

Bighetti EJB, Souza-Brito ARM, Faria EC, Oliveira HCF 2004. Chronic treatment with bark infusion from Croton cajucara lowers plasma triglyceride levels in genetic hyperlipidemic mice. Can J Physiol Pharmacol 82: 387-392.

Carvalho JCT, Silva MFC, Maciel MAM, Pinto AC, Nunes DS, Lima RM, Bastos JK, Sarti SJ 1996. Investigation of anti-inflammatory and antinociceptive activities prototype of trans-dehydrocrotonin, a 19-norclerodane diterpene from Croton cajucara. Part 1. Planta Med 62: 402-404.

Corrêa DHA, Melo PS, Carvallho CAA, Azevedo MBM, Durán N, Haun M 2005. Dehydrocrotonin and its betacyclodextrin complex: Cytotoxicity in V79 fibroblasts and rat cultured hepatocytes. Eur J Pharmacol 510: 17-24.

Farias RAF, Rao VSN, Viana GSB, Silveira ER, Maciel MAM, Pinto AC 1997. Hypoglycemic effect of transdehydrocrotonin, a nor-clerodane diterpene from Croton cajucara. Planta Med 63: 558-560.

Feng SS, Ruan G, Li QT 2004. Fabrication and characterizations of a novel drug delivery device liposomes-inmicrosphere (LIM). Biomaterials 25: 5181-5189.

Freire ACG, Assis CF, Frick AO, Melo PS, Haun M, Aoyama H, Durán N, Sauer MM, Kállas EG, Ferreira CV 2003. Influence of protein phosphatase inhibitors on HL60 cells death induction by dehydrocrotonin. Leukemia Res 27: 823-829.

Giordano OS, Pestchanker MJ, Guerreiro E, Saad JR, Enriz RD, Rodriguez AM, Jáuregui EA, Guzmán J, Maria AOM, Wendel GH 1992. Structure-activity relationship in the gastric cytoprotective effect of several sesquiterpene lactones. J Med Chem 35: 2452-2458.

Grynberg NF, Echevarria A, Lima JE, Pamplona SSR, Pinto AC, Maciel MAM 1999. Anti-tumour activity of two 19-nor-clerodane diterpenes, trans-dehydrocrotonin and trans-crotonin, from Croton cajucara. Planta Med 65: 687-689.
Gupte A, Ciftci K 2004. Formulation and characterization of Paclitaxel, 5-FU and Paclitaxel + 5-FU microspheres. Int J Pharm 276: 93-106.

Hiruma-Lima CA, Spadari-Bratfisch RC, Grassi-Kassisse DM, Souza-Brito ARM 1999. Antiulcerogenic mechanisms of dehydrocrotonin, a diterpene lactone obtained from Croton cajucara. Planta Med 65: 325-330.

Hiruma-Lima CA, Gracioso JS, Rodríguez JA, Haun M, Nunes DS, Souza-Brito ARM 2000. Gastroprotective effect of essential oil from Croton cajucara Benth. (Euphorbiaceae). J. Ethnopharmacol 69: 229-234.

Hiruma-Lima CA, Toma W, Gracioso JS, Almeida ABA, Batista LM, Magri L, Paula ACB, Soares FR, Nunes DS, Souza-Brito ARM 2002. Natural trans-crotonin: The antiulcerogenic effect of another diterpene isolated from the bark of Croton cajucara Benth. Biol Pharm Bull 25: 452-456.

Jin Y, Li J, Rong LF, Li YH, Guo L, Xu SY 2005. Antihepatocarcinoma effects of 5-fluorouracil encapsulated by galactosylceramide liposomes in vivo and in vitro. World J Gastroenterol 11: 2643-2646.

Kim BY, Jeong JH, Park K, Kim JD 2005. Bioadhesive interaction and hypoglycemic effect of insulin-loaded lectin-microparticle conjugates in oral insulin delivery system. J Control Release 102: 525-538.

Kupchan SM, Eakin MA, Thomas AM, 1971. Tumor inhibitors. 69. Structure-cytotoxicity relations among the sesquiterpene lactones. J Med Chem 14: 1147-1152.

Le Ray AM, Gautier H, Laty MK, Daculsi G, Merle C, Jacqueline C, Hamel A, Caillon J 2005. In vitro and in vivo bactericidal activities of vancomycin dispersed in porous biodegradable poly (epsilon-caprolactone) microparticles. Antimicrob Agents Chemother 49: 3025-3027.

Luna Costa AM, Sila JCR, Campos AR, Rao VSN, Maciel MAM, Pinto AC 1999. Antioestrogenic effect of trans-dehydrocrotonin, a nor-clerodane diterpene from Croton cajucara Benth. in rats. Phytother Res 13: 689-691.

Maciel MAM, Pinto AC, Brado SN, Arruda AC 1998a. Estudo da variação dos teores de terpenóides bioativos isolados das cascas do caule de Croton cajucara, nativos e cultivados no estado do Pará. Revista Universidade Rural, Série Ciências Exatas e da Terra, 18/20: 17-34. (http://www.ufrrj.br/edit/revista.htm)

Maciel MAM, Pinto AC, Brabo SN, Silva MN 1998b. Terpenoids from Croton cajucara. Phytochemistry 49: 823-828.

Maciel MAM, Pinto AC, Arruda AC, Pamplona SGSR, Vanderlinde FA, Lapa AJ, Echevarria A, Grynberg NF, Cólus IMS, Farias RAF, Costa AML, Rao VSN 2000. Ethnopharmacology, phytochesmistry and pharmacology: a successful combination in the study of Croton cajucara. J. Ethnopharmacol. 70: 41-55.

Maciel MAM, Pinto AC, Veiga Jr VF, Martins JR, Grynberg NF, Echevarria A, Lapa AJ, Vanderlinde FA 2002a. Croton cajucara as an alternative to traditional medicine in a modern health system. In: Phytochem Pharmacol II 
Ser Recent Prog Med Plants 8: 459-475.

Maciel MAM, Pinto AC, Veiga Jr VF 2002b. Plantas Medicinais: a necessidade de estudos multidiscilplinares. Quim Nova 25: 429-438.

Maciel MAM, Pinto AC, Kaiser CR 2003. NMR and structure review of some natural furoclerodanes. Magn Reson Chem 41: 278-282.

Maciel MAM, Cortez JKPC, Gomes FES 2006. O gênero Croton e aspectos relevantes de diterpenos clerodanos. Revista Fitos 2: 54-73.

Maciel MAM, Martins JR, Pinto AC, Kaiser CR, Esteves-Souza A, Echevarria A 2007. Natural and semi-synthetic clerodanes of Croton cajucara and their cytotoxic effects against Ehrlich carcinoma and human k562 leukemia cells. J Braz Chem Soc 18: 391-396.

Maillard S, Ameller T, Gauduchon J, Gougelet A, Gouilleux F, Legrand P, Marsaud V, Fattal E, Sola B, Renoir JM 2005. Inovative drug delivery nanosystems improve the anti-tumour activity in vitro and in vivo of anti-estrogens in human breast cancer and multiple myeloma. J Steroid Biochem Mol Biol 94: 111-121.

Melo PS, Durán N, Haun M 2001. Cytotoxicity of derivatives from dehydrocrotonin on V79 cells and Escherichia coli. Toxicology 159: 135-141.

Melo PS, Durán N, Hiruma-Lima CA, Souza-Brito ARM, Haun M 2003. Comparison of the gastroprotective effect of a diterpene lactone isolated from Croton cajucara with its synthetic derivatives. J Ethnopharmacol 87: 169-174.

Melo PS, Justo GZ, Durán N, Haun M 2004. Natural killer cell activity and anti-tumour effects of dehydrocrotonin and its synthetic derivatives. Eur J Pharmacol 487: 47-54.

Memisoglu-Bilensoy E, Vural I, Bochot A, Renoir JM, Duchene D, Hincal AA 2005. Tamoxifen citrate loaded amphiphilic beta-cyclodextrin nanoparticles: in vitro characterization and cytotoxicity. J Control Release 104: 489-496.

Mourão SC, Costa PI, Salgado HRN, Gremião MPD 2005. Improvement of antischistosomal activity of praziquantel by incorporation into phosphatidylcholine-containing liposomes. Int $J$ Pharm 295: 157-162.

Mullins DW, Walker TM, Burger CJ, Elgert KD 1997. Taxolmediated changes in fibrosarcoma-induced immune cell function: modulation of antitumor activities. Cancer Immunol Immunother 45: 20-28.

Nakayachi T, Yasumoto E, Nakano K, Morshed SRM, Hashimoto K, Kikuchi H, Nishikawa H, Kawase M, Sakagami H 2004. Structure-activity relationships of $\alpha, \beta$-unsaturated ketones as assessed by their cytotoxicity against oral tumor cells. Anticancer Res 24: 737-742.

Pax F, Hoffmann H 1931. Euphorbiaceae. In: Engler A, Prantil K (org.) Die natürlichen pflanzenfamilien. 2.ed. 19C. Leipzig: Engelmann, p.11-23.

Ratner BD 2002. Reducing capsular thickness and enhancing angiogenesis around implant drug release systems. $J$ Control Release 78: 211-218.

Ravi-Kumar MNV 2000. Nano and microparticles as controlled drug delivery devices. J Pharm Sci 3: 234-258.

Ribeiro-Costa RM, Alves AJ, Santos NP, Nascimento SC, Gonçalves ECP, Silva NH, Honda NK, SantosMagalhães NS 2004. In vitro and in vivo properties of usnic acid encapsulated into PLGA-microspheres. J Microencap 21: 371-384.

Rodríguez JA, Haun M 1999. Cytotoxicity of transdehydrocrotonin from Croton cajucara on V79 Cells and rat hepatocytes. Planta Med 65: 522-526.

Rodríguez JA, Hiruma-Lima CA, Souza-Brito ARM 2004. Antiulcer activity and subacute toxicity of transdehydrocrotonin from Croton cajucara. Hum Exp Toxicol 23: 455-461.

Silva RM, Santos FA, Rao VSN, Maciel MAM, Pinto AC 2001a. Blood glucose- and triglyceride-lowering effect of trans-dehydrocrotonin, a diterpene from Croton cajucara Benth., in rats. Diab Obes Metab 3: 452-456.

Silva RM, Santos FA, Maciel MAM, Pinto AC, Rao VSN 2001b. Effect of trans-dehydrocrotonin, a 19-nor-clerodane diterpene from Croton cajucara on experimental hypertriglyceridaemia and hypercholesterolaemia induced by triton WR1339 (tyloxapol) in mice. Planta Med 67: 763-765.

Silva RM, Santos FA, Rao VSN, Maciel MAM, Pinto AC 2001c. The lipid-lowering effect of trans-dehydrocrotonin, a clerodane diterpene from Croton cajucara Benth. in mice fed on high-fat diet. J Pharm Pharmacol 53: 535-539.

Silva RM, Oliveira FA, Cunha KMA, Maia JL, Maciel MAM, Pinto AC, Nascimento NRF, Santos FA, Rao VSN 2005. Cardiovascular effects of trans-dehydrocrotonin, a diterpene from Croton cajucara in rats. Vasc Pharmacol 43: 11-18.

Simões CMO, Schenkel EP, Gosmann G, Mello JCP, Mentz L, Petrovick PR 2004. Farmacognosia da Planta ao Medicamento. 5.ed. Porto Alegre/Florianópolis: Editora da UFSC/Editora UFRGS.

Soares MCP 2004. Would sacaca, Croton cajucara Benth (Euphorbiaceae) be an hepatotoxic plant like Germander, Teucrium chamaedrys L. (Labiatae)? Rev Soc Bras Med Trop 37: 96-97.

Souza MAA, Souza SR, Veiga Jr VF, Cortez JKPC, Leal RS, Dantas TNC, Maciel MAM 2006. Composição química do óleo fixo de Croton cajucara e determinação das suas propriedades fungicidas. Rev Bras Farmacogn 16(Supl.): 599-610.

Souza-Brito ARM, Rodríguez JA, Hiruma-Lima CA, Haun M, Nunes DC 1998. Antiulcerogenic activity of transdehydrocrotonin from Croton cajucara. Planta Med 64: 126-129.

Teixeira M, Alonso MJ, Pinto MMM, Barbosa CM 2005. Development and characterization of PLGA nanospheres and nanocapsules containing xanthone 
and 3-methoxyxanthone. Eur. J Pharm Biopharm 59:

491-500.

Veiga Jr VF, Pinto AC, Maciel MAM 2005. Medicinal plants: Safe cure? Quim Nova 28: 519-528.

Wattenberg L 1995. Chalcones, myo-inositol and other novel inhibitors of pulmonary carcinogenesis. $J$ Cell Biochem 22: 162-168. 